\title{
Quantitative Radiographic Evaluation of Elbow Incongruity in Labrador and Golden Retrievers with Confirmed Medial Coronoid Disease
}

\author{
Ayman Mostafa ${ }^{1}$ Ingo Nolte ${ }^{2}$ Patrick Wefstaedt ${ }^{2}$ \\ ${ }^{1}$ Department of Small Animal Surgery and Radiology, College of \\ Veterinary Medicine, Cairo University, Giza, Egypt \\ 2 Small Animal Hospital, University of Veterinary Medicine, Hannover, \\ Foundation, Hannover, Germany
}

\begin{abstract}
Address for correspondence Patrick Wefstaedt, DVM, Small Animal Hospital, University of Veterinary Medicine, Hannover, Foundation, Bünteweg 9, 30559 Hannover, Germany (e-mail: Wefstaedt@googlemail.com).
\end{abstract}

Vet Comp Orthop Traumatol 2019;32:10-17.

\begin{abstract}
Objectives The purpose of this study was to objectively estimate humeroradial (HR), humeroulnar (HU) and radioulnar (RU) congruity on mediolateral elbow radiographs of Labrador and Golden Retrievers without and with medial coronoid disease (MCD), and to determine the correlation between traditional and modified RU-step assessment techniques. Materials and Methods Extended mediolateral elbow radiographs of Labrador and Golden Retrievers without MCD (control group) and with confirmed MCD (diseased group) were investigated. Absolute and average $\mathrm{HR}$ and $\mathrm{HU}$ distances were determined and standardized by the radius of the corresponding humeral condyle. Traditional RUstep was measured, and a modified procedure of RU-step assessment was generated. The correlation between the two RU-step assessment procedures was tested.

Results A total of 131 (197 elbows) Labrador and Golden Retrievers met the criteria for inclusion in the control and diseased groups. The normalized HR and HU distances increased significantly $(p \leq 0.002)$ in dogs with MCD. There was a significant increase

Keywords

- elbow dysplasia

- medial coronoid disease

- radiography

- osteoarthritis

- canine $(p<0.0001)$ in the traditional and modified RU-step in dogs with MCD. A significant correlation $\left(r_{\mathrm{s}}=0.74, p<0.0001\right)$ was identified between traditional and modified RU-step calculated for control and diseased elbows.

Clinical Relevance Dogs with confirmed MCD had quantitative radiographic evidences of elbow incongruity. Modified RU-step procedure may be an alternative to the traditional technique and can be utilized during routine quantification of $\mathrm{HR}$ and $\mathrm{HU}$ congruity. Validation of the reported measurements is, however, warranted.
\end{abstract}

\section{Introduction}

Hereditary diseases of canine elbow most commonly involve medial coronoid disease (MCD), ununited anconeal process, osteochondritis of the medial humeral condyle, articular cartilage anomaly and joint incongruity. ${ }^{1-4}$ Medial coronoid disease and associated elbow dysplasia are common developmental problems resulting in thoracic limb lameness in young, large and giant breed dogs, ${ }^{5-7}$ with secondary osteoarthritis being a common sequela in older ages. ${ }^{5,8,9}$ Labrador Retrievers have been reported to be the most common affected breed with MCD and elbow incongruity. $5,7,10$

Elbow incongruity is anatomical misalignment of the articular surfaces of humeroradial (HR), radioulnar (RU) and/ or humeroulnar (HU) joints. ${ }^{11,12}$ Incongruity of the canine elbow plays a potential role in the pathogenesis of MCD and development of associated elbow dysplasia and secondary received

February 8, 2018

accepted after revision

July 20, 2018 (c) 2019 Georg Thieme Verlag KG

Stuttgart · New York
DOI https://doi.org/

10.1055/s-0038-1672210. ISSN 0932-0814. 
osteoarthritis. ${ }^{13-17}$ Medial coronoid disease may develop due to the undergrowth of the radius (short radius) relative to the ulna (RU incongruity). ${ }^{1,10}$ The RU incongruity results in an altered loading pattern on the medial compartment of the elbow and subsequent cartilage pathology and fragmentation of the corresponding medial coronoid process. ${ }^{15,18}$ The resultant RU incongruity may also be consistent with the development of HR incongruity due to the relative increase in the HR joint space. Furthermore, underdevelopment of the ulnar trochlear notch and associated decrease in its radius of curvature has been reported to be another possible cause of MCD in dogs. ${ }^{15-17}$ The underdeveloped ulnar trochlear notch, with respect to the corresponding radius and humeral condyle, may then result in RU and HU incongruity, respectively, and subsequent increased load on the medial coronoid process and anconeal process. ${ }^{15,17}$ However, a relative increase in the radius of curvature of the ulnar trochlear notch was evidenced in dogs with elbow dysplasia and osteoarthritis to accommodate the corresponding humeral condyle in these patients. ${ }^{16,17}$ Overall, misshapen ulnar trochlear notch and undergrown (short) radius may be consistent with $\mathrm{HU}$, RU and HR incongruity.

To the authors' knowledge, evaluation of HR, HU and RU incongruity has so far not been quantified collectively on a lateral elbow radiograph with confirmed MCD. Furthermore, no modification of the traditional RU-step measurement has previously been reported to assess RU incongruity in dogs. Quantification of elbow incongruity may be essential to achieve early diagnosis and, thus, better prognosis of MCD to help develop efficient treatment plans in the future. ${ }^{19,20}$ The objective of the study was therefore to quantitatively determine the incongruity of $\mathrm{HR}, \mathrm{HU}$ and RU joints on the extended mediolateral elbow radiograph for Labrador and Golden Retrievers confirmed to have MCD. The study also aimed to generate a modified radiographic procedure for assessment of RU-step and determine its correlation with the conventional method. The study hypotheses were (1) HR, HU and/or RU incongruity most likely develops in Labrador and Golden Retrievers with confirmed MCD; (2) modified RU-step assessment procedure would be correlated with the conventional method and could then be used as an alternative technique for the radiographic evaluation of canine RU-step.

\section{Materials and Methods}

\section{Dogs}

Medical records and elbow radiographs of Labrador and Golden Retrievers without (controls) and with confirmed unilateral or bilateral MCD (diseased group) were retrieved and reviewed for inclusion criteria. Clinically affected dogs with or without radiographic evidences of $\mathrm{MCD}$, that was confirmed via computed tomography (CT), arthroscopy or at the time of surgery, were included. Dogs that had MCD along with other elbow abnormality (such as ununited anconeal process, osteochondritis of the medial humeral condyle or ununited medial humeral epicondyle) or marked arthrosis were excluded. The control group involved Labrador and Golden Retrievers that had no history or clinical signs of elbow disease, and for which radiographic examinations of elbow joints revealed no abnormalities. Dogs less than 7 months old were selected as controls because individuals that reach this age without clinical or radiographic evidence of MCD are unlikely to have such a disease. This is because clinical signs of MCD have previously been reported as early as 12 to 15 weeks of age, ${ }^{21,22}$ and radiographic signs were not evidenced until 7 or 8 months old. ${ }^{23-26}$ The age, body weight and sex were recorded for all enrolled dogs. The study protocol was approved by the Scientific Committee of the Small Animal Hospital at the University of Veterinary Medicine, Foundation, Hanover, Germany. Ethical approval was not necessary as elbow radiographs were acquired as a routine database for all enrolled dogs.

\section{Radiographic Assessment}

Extended mediolateral radiographs of elbow joints were obtained to quantify the congruity of normal elbows and those with confirmed MCD. Positioning was judged as satisfactory if medial and lateral humeral condylar silhouettes were concentric. Projections that allowed improper outlining of the articular margin of the radial head or the ulnar trochlear notch (due to superimposition by humeral condyle) were excluded. All radiographs were digitized and approved in terms of quality and positioning by a qualified radiologist (IN) before investigation.

Elbow joint congruity was objectively determined via calculating the absolute, average and normalized values of HR and HU distances. The humeroradial distances (HRD) represent the absolute distances between the centre of rotation of the humeral condyle and proximocranial, central and proximocaudal points created on the fovea of the radial head (cranial, central and caudal HRD, - Fig. 1). The

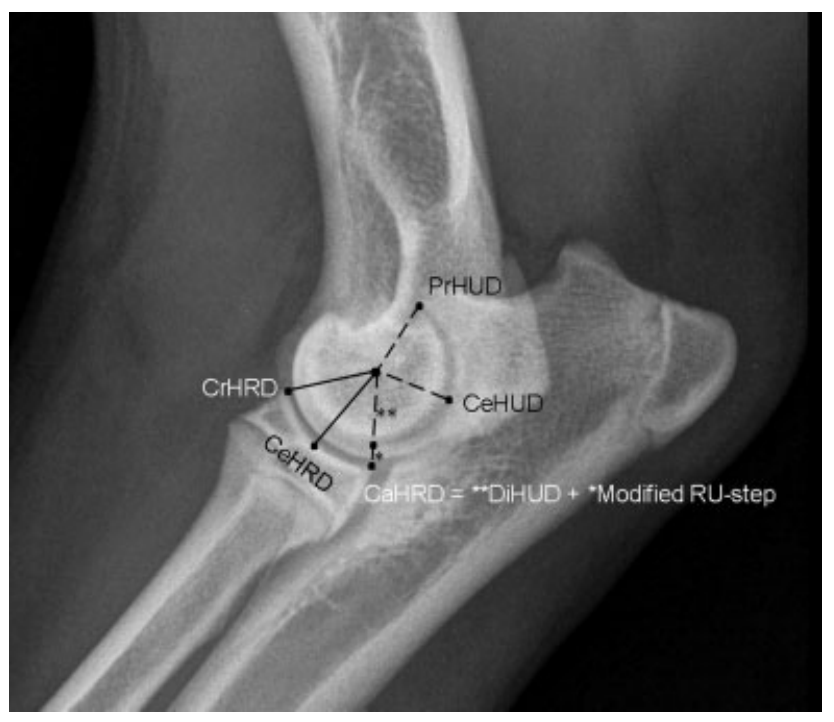

Fig. 1 Mediolateral elbow radiograph of a Labrador Retriever confirmed to have medial coronoid disease illustrating measurements of humeroradial and humeroulnar distances and modified radioulnar step. CrHRD, cranial humeroradial distance; CeHRD, central humeroradial distance; CaHRD, caudal humeroradial distance; PrHUD, proximal humeroulnar distance; CeHUD, central humeroulnar distance; DiHUD, distal humeroulnar distance; RU-step, radioulnar step. Note the subtrochlear ulnar sclerosis associated with the elbow. * DiHUD; ** modified RU-step. 
humeroulnar distances (HUD) represent the absolute distances between the centre of rotation of the humeral condyle and proximal, central and distal points created along the medial margin of the ulnar trochlear notch (proximal, central and distal HUD, - Fig. 1). The proximal point of the ulnar trochlear notch was located on the distocranial edge of the anconeal process. The distal point of the ulnar trochlear notch was defined as the point of intersection between the medial margin of the ulnar trochlear notch (at the level of the base of the medial coronoid process) ${ }^{12,14,18,27}$ and the line drawn to measure the caudal HRD (the line connecting the centre of rotation of the humeral condyle and the proximocaudal extent of the radial head) ( - Fig. 1). The average value of each set of HRD and HUD was calculated to determine the overall distance between the centre of rotation of humeral condyle and each of the fovea of the radial head and ulnar trochlear notch, respectively. To alleviate the interindividual size variations, the absolute and average values of each distance were standardized by dividing these values by the radius of the corresponding humeral condyle.

Radioulnar congruity was quantified by assessment of RUstep. The RU-step was traditionally evaluated by measuring the vertical distance between a line connecting the proximocranial and proximocaudal extents of the fovea of the radial head and a parallel line drawn along the lateral coronoid process (traditional RU-step, - Fig. 2) ${ }^{27-30}$ A modified procedure for assessing RU-step (modified RU-step) was generated in the current study by subtraction of the absolute value of the distal HUD from that of the caudal HRD. The difference between the two distances appears to represent the step between the proximocaudal extent of the radial head and the distal extent of the medial margin of the ulnar trochlear notch at the level of the base of the MCP. All measurements were performed by use of a free medical and radiological processing software (ImageJ 1.41/Java 1.6.0_21) previously utilized in veterinary orthopaedic research. ${ }^{31,32} \mathrm{~A}$ magnifica-

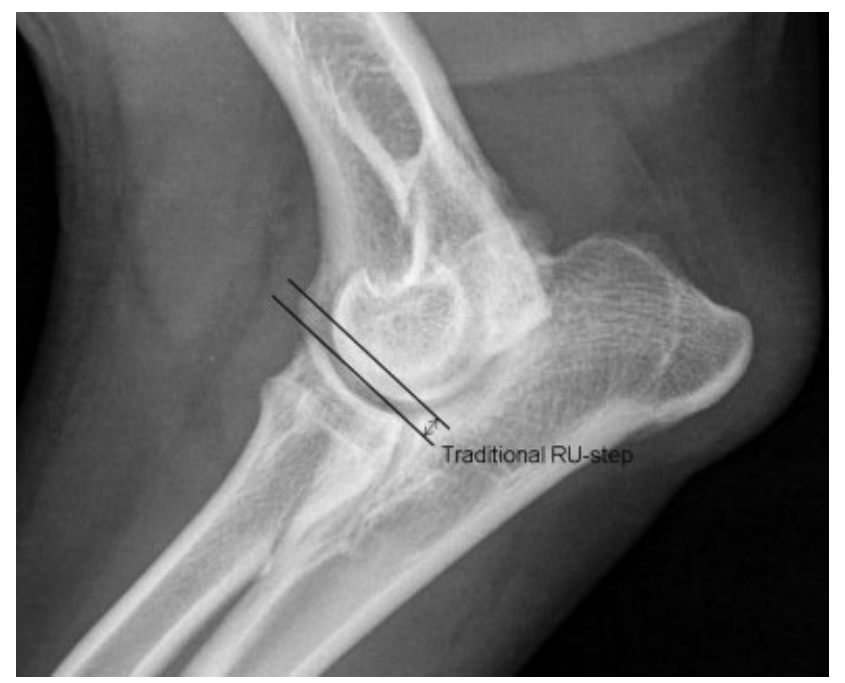

Fig. 2 Mediolateral elbow radiograph of a Golden Retriever confirmed to have medial coronoid disease illustrating measurement of traditional radioulnar step. Note the degenerative changes associated with elbow joint. RUstep, radioulnar step. tion of 300 was used to measure the HRD and HUD, as well as the radius of the humeral condyle, whereas a higher magnification of 800 was used to measure the traditional RU-step.

\section{Statistical Analysis}

Before statistical analysis, most of the data were proven to be normally distributed by the use of D'Agostino and Pearson omnibus test. Despite that some of our numerical data did not follow the normal distribution, we adopted the central limit theorem, ${ }^{33}$ and used parametric tests for data analysis. Selected variables were reported as means with standard deviations, and significance was set at $p$-value $<0.05$. The means of the absolute, average and normalized values of HRD and HUD, as well as the traditional and modified RU-step calculated for elbows with confirmed MCD, were compared with those calculated for control elbows. Variables were compared with an unpaired, twotailed $t$-test by the use of GraphPad Prism software (GraphPad Prism version 6.00, La Jolla, California, United States), and a 95\% confidence interval (CI) was calculated for each selected variable. A Spearman correlation coefficient $\left(r_{s}\right)$ and $R^{2}$ value were calculated to determine the relationship between the traditional and modified radiographic techniques of RU-step assessment.

\section{Results}

\section{Dogs}

A total of 70 dogs (108 elbows), (53 Labrador Retrievers and 17 Golden Retrievers), met the criteria for inclusion in the group of patients with confirmed MCD. Among these 70 patients, 38 (31 Labrador Retrievers and 7 Golden Retrievers) had bilateral MCD and 32 (22 Labrador Retrievers and 10 Golden Retrievers) had unilateral disease. A total of 61 dogs (89 elbows), 44 Labrador Retrievers and 17 Golden Retrievers, met the criteria for inclusion in the control group. The contralateral thoracic limbs of 20 Labrador Retrievers and 9 Golden Retrievers diagnosed with unilateral MCD were considered as controls, as they had no clinical or radiographic evidence of elbow abnormality on that contralateral side. Twelve Labrador Retrievers and five Golden Retrievers were admitted for routine radiographic examination of the thoracic limbs following acute trauma and showed no evidence of orthopaedic abnormalities. Six Labrador Retrievers were admitted with distal humeral or proximal radial and/or ulnar fracture with no elbow disease. Three Labrador Retrievers and two Golden Retrievers had hip dysplasia and/or intervertebral disc disease with no clinical or radiographic evidence of elbow abnormality. The control group also involved three Labrador Retrievers and a Golden Retriever with shoulder, carpal or digital affections and healthy elbow joints. All control limbs belonged to dogs that reached 7 months of age without history or current clinical or radiographic evidence of elbow abnormality. The age and body weight did not differ between the control and diseased groups of dogs. Male dogs were more than twice as frequent as female dogs (male-to-female ratio $=2.2: 1$ ) in both groups. The results of age and body weight analysis and sex distribution are demonstrated in - Table $\mathbf{1}$. 
Table 1 Mean ( \pm SD) values and ranges of the age and body weight and sex distribution for Labrador Retrievers and Golden Retrievers without (control group) and with (diseased group) medial coronoid disease

\begin{tabular}{|l|l|l|l|l|l|}
\hline \multirow{2}{*}{ Parameter } & \multicolumn{2}{l|}{$\begin{array}{l}\text { Control group } \\
\text { (61 dogs, 89 elbows) }\end{array}$} & \multicolumn{2}{l|}{$\begin{array}{l}\text { Diseased group } \\
\text { (70 dogs, 108 elbows) }\end{array}$} & -Value \\
\cline { 2 - 7 } & Mean \pm SD & Range & Mean \pm SD & Range & \\
\hline Age (years) & $5.4 \pm 3.6$ & $0.6-12.4$ & $4.9 \pm 3.4$ & $0.6-12.4$ & 0.455 \\
\hline Body weight $(\mathrm{kg})$ & $32.0 \pm 5.2$ & $14.5-39.9$ & $33.3 \pm 6.4$ & $20.5-64$ & 0.200 \\
\hline Sex distribution & Male & Female & Male & Female & \\
\cline { 2 - 7 } & $42(11$ castrated $)$ & $19(11$ spayed $)$ & $48(10$ castrated $)$ & $22(12$ spayed $)$ & \\
\hline
\end{tabular}

Abbreviation: SD, standard deviation.

Note: $p$-Value $<0.05$ compared with controls.

\section{Radiographic Assessment}

The absolute and average values of normalized HR and HU distances increased significantly $(p \leq 0.002)$ in Labrador and Golden Retrievers with confirmed MCD compared with controls (-Table 2). A significant increase $(p<0.0001)$ in the traditional and modified RU-step was identified in dogs with confirmed MCD compared with controls. The 95\% confidence intervals $(\mathrm{CI})$ of the absolute, average and

Table 2 Mean $( \pm S D)$ values and 95\% confidence intervals for radiographic measurements of the absolute, average and normalized values of humeroradial and humeroulnar distances, as well as the traditional and modified radioulnar step, for Labrador and Golden Retrievers without (control group) and with (diseased group) medial coronoid disease

\begin{tabular}{|c|c|c|c|c|c|}
\hline \multirow[t]{2}{*}{ Variable } & \multicolumn{2}{|c|}{$\begin{array}{l}\text { Control group } \\
\text { (61 dogs, } 89 \text { elbows) }\end{array}$} & \multicolumn{2}{|c|}{$\begin{array}{l}\text { Diseased group } \\
\text { ( } 70 \text { dogs, } 108 \text { elbows) }\end{array}$} & \multirow[t]{2}{*}{$p$-Value } \\
\hline & Mean \pm SD & $95 \% \mathrm{Cl}$ & Mean \pm SD & $95 \% \mathrm{Cl}$ & \\
\hline Radius of the humeral condyle $(\mathrm{r}) / \mathrm{mm}$ & $7.7 \pm 0.6$ & $7.6-7.8$ & $7.5 \pm 0.6$ & $7.4-7.7$ & 0.099 \\
\hline \multicolumn{6}{|l|}{ Humeroradial distances (HRD)/mm } \\
\hline Cranial HRD & $10.4 \pm 0.9$ & $10.2-10.6$ & $10.4 \pm 0.9$ & $10.2-10.6$ & 0.689 \\
\hline Central HRD & $10.8 \pm 0.9$ & $10.6-11.0$ & $10.9 \pm 0.9$ & $10.7-11.1$ & 0.602 \\
\hline Caudal HRD & $10.9 \pm 1.0$ & $10.7-11.2$ & $11.4 \pm 1.1$ & $11.1-11.6$ & 0.007 \\
\hline Average HRD & $10.7 \pm 0.9$ & $10.5-10.9$ & $10.9 \pm 0.9$ & $10.7-11.1$ & 0.156 \\
\hline \multicolumn{6}{|l|}{ Humeroradial ratios (normalized HRD) } \\
\hline Cranial HRD:r & $1.35 \pm 0.07$ & $1.33-1.36$ & $1.38 \pm 0.08$ & $1.37-1.40$ & 0.002 \\
\hline Central HRD:r & $1.41 \pm 0.06$ & $1.39-1.42$ & $1.44 \pm 0.07$ & $1.43-1.46$ & 0.0002 \\
\hline Caudal HRD:r & $1.42 \pm 0.08$ & $1.40-1.44$ & $1.51 \pm 0.09$ & $1.49-1.52$ & $<0.0001$ \\
\hline Average HRD ratio & $1.39 \pm 0.06$ & $1.38-1.40$ & $1.44 \pm 0.07$ & $1.43-1.46$ & $<0.0001$ \\
\hline \multicolumn{6}{|l|}{ Humeroulnar distances (HUD)/mm } \\
\hline Distal HUD & $9.1 \pm 0.8$ & $8.9-9.3$ & $9.1 \pm 0.8$ & $9.0-9.3$ & 0.735 \\
\hline Central HUD & $8.8 \pm 0.8$ & $8.6-9.0$ & $9.3 \pm 0.9$ & $9.1-9.4$ & 0.001 \\
\hline Proximal HUD & $8.7 \pm 0.8$ & $8.5-8.8$ & $8.8 \pm 0.9$ & $8.6-9.0$ & 0.350 \\
\hline Average HUD & $8.9 \pm 0.8$ & $8.7-9.0$ & $9.1 \pm 0.8$ & $8.9-9.2$ & 0.078 \\
\hline \multicolumn{6}{|l|}{ Humeroulnar ratios (normalized HUD) } \\
\hline Distal HUD:r & $1.18 \pm 0.04$ & $1.17-1.19$ & $1.21 \pm 0.05$ & $1.20-1.22$ & $<0.0001$ \\
\hline Central HUD:r & $1.15 \pm 0.05$ & $1.14-1.16$ & $1.23 \pm 0.07$ & $1.21-1.24$ & $<0.0001$ \\
\hline Proximal HUD:r & $1.13 \pm 0.06$ & $1.12-1.14$ & $1.17 \pm 0.07$ & $1.15-1.18$ & 0.0002 \\
\hline Average HUD ratio & $1.15 \pm 0.03$ & $1.15-1.16$ & $1.20 \pm 0.05$ & $1.19-1.21$ & $<0.0001$ \\
\hline \multicolumn{6}{|l|}{ Radioulnar step (RU-step)/mm } \\
\hline Traditional RU-step & $1.3 \pm 0.3$ & $1.2-1.4$ & $2.0 \pm 0.5$ & $1.9-2.1$ & $<0.0001$ \\
\hline Modified RU-step & $1.8 \pm 0.6$ & $1.7-1.9$ & $2.2 \pm 0.7$ & $2.1-2.4$ & $<0.0001$ \\
\hline
\end{tabular}

Note: $p$-Value $<0.05$ compared with controls. 
normalized values of HR and HU distances, as well as of the traditional and modified RU-step, are illustrated in - Table 2. There was a significant correlation between the traditional and modified techniques of RU-step assessment calculated for control and diseased elbows $\left(r_{s}=0.74 ; p<0.0001\right.$; $R^{2}=0.53$; modified RUstep $=0.81 \times$ traditional RUstep +0.61 ) (-Fig. 3).

\section{Discussion}

The main findings were: (1) each absolute and average values of normalized HR and HU distances increased significantly $(p \leq 0.002)$ in Labrador and Golden Retrievers with confirmed MCD. Standardized HR and HU distances greater than 1.4 and 1.2 were consistent with HR and HU incongruity, respectively; (2) a significant increase $(p<0.0001)$ in the traditional and modified RU-step was identified in diseased dogs. Traditional and modified RU-step greater than $1.4 \mathrm{~mm}$ and $1.9 \mathrm{~mm}$, respectively, were consistent with RU incongruity; (3) there was a significant correlation between the traditional and modified procedures of RU-step assessment calculated for control and diseased elbows $\left(r_{s}=0.74, p<0.0001\right)$.

Computed tomography and arthroscopy remain the most accurate imaging modalities used for early diagnosis of canine MCD. However, lack of their availability and personal expertise remains a challenge, particularly in veterinary practice. In contrast, radiography is the mainstay for initial diagnosis of elbow disease in patients with thoracic limb lameness, ${ }^{34}$ and is further considered the most available imaging modality widely utilized in clinical practice. Furthermore, the previously reported good sensitivity and specificity of radiography on determining elbow incongruity makes it a valuable imaging modality to screen canine elbows for radiographic evidence of incongruity. ${ }^{11,13}$ However, definitive diagnosis of MCD using radiography is another challenge in young growing dogs, most likely those less than 7 months of age. ${ }^{21}$ Radiographic diagnosis of MCD

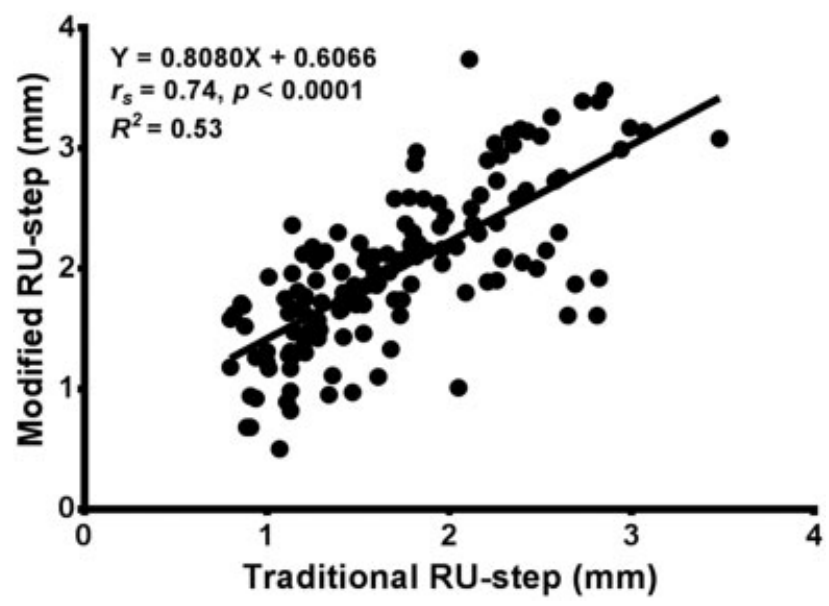

Fig. 3 Scatter plots of traditional versus modified RU-step determined from mediolateral elbow radiographs of Labrador Retrievers and Golden Retrievers without and with medial coronoid disease. The solid line represents the linear equation fitted to the data points. The associated equation is provided. RUstep, radioulnar step. relies initially on the changes associated with accompanied elbow dysplasia and osteoarthritis in patients older than 7 months of age. $8,15,21,35,36$ Based on previous studies, clinical manifestation of MCD has been reported as early as 3 months of age, and 15 weeks of age in a different study, 21,22 whereas radiographic changes associated with MCD were not identified before 7 months of age. ${ }^{23-26}$ Based on that, the control subjects enrolled in the present study were selected to be older than 7 months of age. Nonetheless, the absence of clinical and radiographic signs of elbow disease in Labrador and Golden Retrievers more than 7 months old does not completely rule out the occurrence of MCD in our controls. Although this is a major caveat of the study, the authors believe that it would not be practical to utilize an invasive or expensive imaging modality (i.e. arthroscopy or CT) to only rule out the occurrence of the disease in clinically and radiographically normal elbows of dogs older than 7 months of age.

There is a certain degree of physiological geometric elbow incongruity in dogs; ${ }^{37}$ however, pathological form of incongruity is usually consistent with elbow dysplasia and secondary osteoarthritis. ${ }^{14}$ The limited physiological HR, $\mathrm{HU}$ and RU incongruity associated with normal elbows quantified in the present study may be consistent with the normal geometry of subchondral bone margins and joint spaces of the corresponding articulations that was previously reported. ${ }^{38}$ Incongruity of canine elbow is less likely to be detected at the time of MCD diagnosis; ${ }^{13,17,39}$ thus, early quantification of the pathological form of $\mathrm{HR}, \mathrm{HU}$ and RU incongruity is expected to be of value in veterinary clinical practice. The pathological HR and RU incongruity associated with MCD is most likely consistent with short radius syndrome. ${ }^{13,14}$ Further, HU incongruity and corresponding MCD commonly develop due to a relatively low curvature radius and deformed trochlear notch of the ulna with respect to the corresponding humeral trochlea. ${ }^{15-17}$

The compound nature of elbow joint may indicate the importance of evaluation of the congruity of each associated articulation (i.e. HR, HU and RU articulations). Different imaging modalities have widely been utilized to assess RU incongruity in dogs through measuring the corresponding RUstep. ${ }^{11,13,15,27,29,30,40-44}$ Humeroulnar incongruity has previously been quantified via calculating the corresponding $\mathrm{HU}$ index on the mediolateral radiograph of canine elbow. ${ }^{27}$ In this previous report, HU subluxation index increased significantly in Labrador Retrievers with MCD, indicating HU incongruity. ${ }^{27}$ Other studies determined HR and HU congruity through evaluating the interosseous gaps and joint spaces of the corresponding articulations on radiography, ${ }^{11,45,46}$ $\mathrm{CT}^{41,47,48}$ and magnetic resonance imaging. ${ }^{37}$ The current study provides the first evidence of radiographic quantification of elbow congruity via calculating the absolute and average HR and HU distances, at three different levels along each corresponding subchondral bone margin, with respect to the radius of associated humeral condyle. The reported HU measurements are expected to be of value in future assessment of the radius of curvature of ulnar trochlear notch and thus monitoring the development of its deformity. The study 
also evaluated RU congruity by determining the traditional RU-step, as well as by creating a modified procedure of RU-step assessment using the normalized distances that were measured to quantify HR and HU congruity. In the present study, elbows confirmed to have MCD showed HR, HU and RU incongruity based on the relative increases in standardized HR and HU distances as well as traditional and modified RUstep, when compared with control elbows. These results are consistent with previous studies that reported greater HU and RU incongruity in dogs with MCD. ${ }^{27,39}$ Incongruity of HU joint was previously evidenced by the greater value of HU subluxation index calculated on the lateral elbow radiographs of Labrador Retrievers with MCD. ${ }^{27}$ However, to the authors' knowledge, no previous reports have quantified the incongruity of HR joint on elbow radiographs of dogs with confirmed MCD. Therefore, the current study evaluated HR incongruity and reported a significant increase in the absolute and average values of normalized HR distances calculated for diseased elbow.

In the study reported here, there was a significant increase $(p<0.0001)$ in traditional and modified RU-step determined on the lateral elbow radiographs of Labrador and Golden Retrievers with confirmed MCD. The mean traditional RU-step measured for control and diseased elbows were $1.3 \mathrm{~mm}$ and $2.0 \mathrm{~mm}$ in the present study, compared with $0.4 \mathrm{~mm}$ and $1.5 \mathrm{~mm}$, respectively, in a previous study by Proks and colleagues. ${ }^{27}$ The relatively increased values of physiological and pathological RU incongruity identified in the present study could be attributed to the older (average, 5.2 years) and larger (131 dogs, 197 elbows) populations of 'normal' and diseased dogs investigated here, compared with the age (average, 1.4 years) and number (51 dogs, 92 elbows) of Labrador Retrievers enrolled in the previous study. ${ }^{27}$ Furthermore, the values of traditional RU-step have previously been reported to range from $1.5 \mathrm{~mm}$ to greater than $4 \mathrm{~mm},{ }^{40}$ and in another study, RU-step measured up to $5 \mathrm{~mm},{ }^{1}$ based on the severity of joint incongruity. The magnitude of a RU-step defect was approximately $2 \mathrm{~mm}$ in a series of nine dogs diagnosed with elbow dysplasia. ${ }^{15}$ These findings are relatively consistent with our results, as calculated $95 \% \mathrm{CI}$ of traditional RU-step was $1.9 \mathrm{~mm}$ to $2.1 \mathrm{~mm}$ (range, up to $3.3 \mathrm{~mm}$ ) for diseased elbows, and a RU-step greater than $1.4 \mathrm{~mm}$ has been suggested to be indicative of RU incongruity. The $95 \% \mathrm{CI}$ of modified RU-step was $2.1 \mathrm{~mm}$ to $2.4 \mathrm{~mm}$ (range, up to $3.7 \mathrm{~mm}$ ) for diseased elbows, and a RU-step greater than $1.9 \mathrm{~mm}$ has been suggested to be indicative of RU incongruity. Based on the good correlation between traditional and modified RU-step, the authors would encourage clinical practitioners to use the modified procedure as an alternative to the conventional method while quantifying HR and HU congruity on the lateral elbow radiograph. Despite the higher diagnostic value of CT and arthroscopy in RU-step assessment, ${ }^{40,42,49}$ radiography remains the most available and applicable imaging modality in veterinary clinical practice to identify a traditional, and currently a modified, RU-step defect.

Radiographic quantification of elbow congruity through calculating standardized HR and HU distances and measur- ing RU-step is expected to be utilized in future clinical studies to determine the early changes associated with MCD and to further investigate the role of incongruity in the progression of canine elbow dysplasia. However, lack of determination of intra- and inter-investigator repeatability and reproducibility of our reported measurements is a limitation of the study. A future investigation is, therefore, warranted to validate the reported radiographic procedures. Furthermore, future assessment of the correlation between the reported radiographic procedures and other CT and arthroscopic techniques, used for diagnosis of canine elbow incongruity, is recommended. Despite the essential role of elbow incongruity in the pathogenesis of canine MCD, ${ }^{13-17}$ a full perception of how joint incongruity and associated elbow dysplasia would develop and progress has not been accomplished yet. ${ }^{17}$ Thus, an additional prospective investigation involving a different cohort of dogs may be required to serially monitor the growth and development of elbows of immature subjects without and with medial coronoid disease. In brief, our current and proposed future studies are expected to achieve a definitive further characterization of the prevalence and severity of elbow incongruity and may therefore provide a variety of future treatment options of canine MCD and elbow dysplasia.

\section{Conclusions}

Standardized HR and HU distances increased in Labrador and Golden Retrievers with confirmed MCD compared with controls. Traditional and modified RU-step was greater in diseased dogs compared with controls. Modified RU-step technique correlated with the conventional method and could be used as an alternative procedure to assess RU-step during routine quantification of HR and HU congruity in dogs with and without MCD. It is, therefore, possible to quantify pathological elbow incongruity and properly distinguish it from the physiological form. It is hoped quantitative determination of elbow incongruity will provide key diagnostic and prognostic criteria for future selection of an efficient treatment plan. However, future investigation remains warranted to prove this proposal and to validate the radiographic measurements reported in the present study.

\section{Funding}

This project was financed by the University of Veterinary Medicine, Hannover, Foundation, Germany, and supported in part by a grant from the Bilateral Exchange of Academics and Scientists program by DAAD (Deutscher Akademischer Austauschdienst).

\section{Conflict of Interest}

None.

\section{Authors Contribution}

All authors contributed to conception of study, study design, and acquisition of data and data analysis and interpretation. All authors drafted, revised and approved the submitted manuscript. 


\section{Acknowledgments}

The authors acknowledge Mr. Andreas Koeppen and Dr. Bach Jan-Peter for their assistance with data collection.

\section{References}

1 Samoy Y, Van Ryssen B, Gielen I, Walschot N, van Bree H. Review of the literature: elbow incongruity in the dog. Vet Comp Orthop Traumatol 2006;19(01):1-8

2 Moores AP, Benigni L, Lamb CR. Computed tomography versus arthroscopy for detection of canine elbow dysplasia lesions. Vet Surg 2008;37(04):390-398

3 Peremans K, Vermeire S, Dobbeleir A, et al. Recognition of anatomical predilection sites in canine elbow pathology on bone scans using micro-single photon emission tomography. Vet J 2011;188(01):64-72

4 Michelsen J. Canine elbow dysplasia: aetiopathogenesis and current treatment recommendations. Vet J 2013;196(01):12-19

5 Fitzpatrick N, Smith TJ, Evans RB, Yeadon R. Radiographic and arthroscopic findings in the elbow joints of 263 dogs with medial coronoid disease. Vet Surg 2009;38(02):213-223

6 Vermote KA, Bergenhuyzen AL, Gielen I, van Bree H, Duchateau L, Van Ryssen B. Elbow lameness in dogs of six years and older: arthroscopic and imaging findings of medial coronoid disease in 51 dogs. Vet Comp Orthop Traumatol 2010;23(01):43-50

7 Coppieters E, Seghers H, Verhoeven G, et al. Arthroscopic, computed tomography, and radiographic findings in 25 dogs with lameness after arthroscopic treatment of medial coronoid disease. Vet Surg 2016;45(02):246-253

8 Keller GG, Kreeger JM, Mann FA, Lattimer JC. Correlation of radiographic, necropsy and histologic findings in 8 dogs with elbow dysplasia. Vet Radiol Ultrasound 1997;38(04):272-276

9 Smith TJ, Fitzpatrick N, Evans RB, Pead MJ. Measurement of ulnar subtrochlear sclerosis using a percentage scale in Labrador Retrievers with minimal radiographic signs of periarticular osteophytosis. Vet Surg 2009;38(02):199-208

10 Temwichitr J, Leegwater PA, Hazewinkel HA. Fragmented coronoid process in the dog: a heritable disease. Vet J 2010;185(02): 123-129

11 Blond L, Dupuis J, Beauregard G, Breton L, Moreau M. Sensitivity and specificity of radiographic detection of canine elbow incongruence in an in vitro model. Vet Radiol Ultrasound 2005;46(03): 210-216

12 Griffon DG. Surgical diseases of the elbow. Chapter 53. In: Tobias KM, Johnston SA, eds. Veterinary Surgery; Small Animal: 2-volume set, 1st ed. Canada: WB Saunders-Elsevier; 2012: 724-759

13 Samoy Y, Gielen I, Saunders J, van Bree H, Van Ryssen B. Sensitivity and specificity of radiography for detection of elbow incongruity in clinical patients. Vet Radiol Ultrasound 2012;53(03):236-244

14 Alves-Pimenta S, Ginja MM, Fernandes AM, Ferreira AJ, MeloPinto P, Colaço B. Computed tomography and radiographic assessment of congruity between the ulnar trochlear notch and humeral trochlea in large breed dogs. Vet Comp Orthop Traumatol 2017;30 (01):8-14

15 Wind AP. Elbow incongruity and developmental elbow disease in the dog: part 1. J Am Anim Hosp Assoc 1986;22:711-724

16 Wind AP. Elbow incongruity and developmental elbow disease in the dog: part II. J Am Anim Hosp Assoc 1986;22:725-730

17 Gemmill TJ, Clements DN. Fragmented coronoid process in the dog: is there a role for incongruency? J Small Anim Pract 2007;48 (07):361-368

18 Alves-Pimenta S, Ginja MM, Colaço J, Fernandes AM, Melo-Pinto P, Colaço B. Curvature radius measurements from the ulnar trochlear notch in large dogs. Anat Rec (Hoboken) 2015;298(10): 1748-1753

19 Thomson MJ, Robins GM. Osteochondrosis of the elbow: a review of the pathogenesis and a new approach to treatment. Aust Vet J 1995;72(10):375-378
20 Ness MG. Treatment of fragmented coronoid process in young dogs by proximal ulnar osteotomy. J Small Anim Pract 1998;39 (01):15-18

21 Lau SF, Wolschrijn CF, Hazewinkel HA, Siebelt M, Voorhout G. The early development of medial coronoid disease in growing Labrador Retrievers: radiographic, computed tomographic, necropsy and micro-computed tomographic findings. Vet J 2013;197(03):724-730

22 Lau SF, Hazewinkel HA, Grinwis GC, et al. Delayed endochondral ossification in early medial coronoid disease (MCD): a morphological and immunohistochemical evaluation in growing Labrador Retrievers. Vet J 2013;197(03):731-738

23 Berzon JL, Quick CB. Fragmented medial coronoid process: anatomical, clinical, and radiographic considerations with case analyses. J Am Anim Hosp Assoc 1980;16:241-251

24 De Rycke LM, Gielen IM, van Bree H, Simoens PJ. Computed tomography of the elbow joint in clinically normal dogs. Am J Vet Res 2002;63(10):1400-1407

25 Meyer-Lindenberg A, Langhann A, Fehr M, Nolte I. Prevalence of fragmented medial coronoid process of the ulna in lame adult dogs. Vet Rec 2002;151(08):230-234

26 Tromblee TC, Jones JC, Bahr AM, Shires PK, Aref S. Effect of computed tomography display window and image plane on diagnostic certainty for characteristics of dysplastic elbow joints in dogs. Am J Vet Res 2007;68(08):858-871

27 Proks P, Necas A, Stehlik L, Srnec R, Griffon DJ. Quantification of humeroulnar incongruity in Labrador Retrievers with and without medial coronoid disease. Vet Surg 2011;40(08):981-986

28 Brunnberg L, Viehmann B, Waibl H. Computergestützte Auswertung von Röntgenbildern zur Erfassung von Parametern der Ellbogengelenksdysplasie-Teil 2 Stufenbildungen im Gelenk (Computer-assisted evaluation of X-ray images to record parameters of elbow dysplasia-Part 2 Stage formations in the joint). Kleintierpraxis 1999;44:637-646

29 Mostafa AA, Nolte I, Wefstaedt P. The prevalence of medial coronoid disease is high in lame breed dogs and quantitative radiographic assessments contribute to the diagnosis. Vet Radiol Ultrasound 2018;59(05):516-528

30 Griffon DJ, Mostafa AA, Blond L, Schaeffer DJ. Radiographic, computed tomographic, and arthroscopic diagnosis of radioulnar incongruence in dogs with medial coronoid disease. Vet Surg 2018;47(03):333-342

31 Mostafa AA, Drüen S, Nolte I, Wefstaedt P. Radiographic evaluation of early periprosthetic femoral bone contrast and prosthetic stem alignment after uncemented and cemented total hip replacement in dogs. Vet Surg 2012;41(01):69-77

32 Mostafa AA, Lucas K, Nolte I, Wefstaedt P. Radiographic evaluation of early periprosthetic acetabular bone contrast and prosthetic head acetabular coverage after uncemented and cemented total hip prosthesis in dogs. BMC Vet Res 2016;12(01):271-279

33 Daniel WW, Cross CL. Biostatistics. A Foundation for Analysis in Health Science. 10th ed. United States: John Wiley \& Sons Inc.; 2013

34 Fitzpatrick N, Yeadon R. Working algorithm for treatment decision making for developmental disease of the medial compartment of the elbow in dogs. Vet Surg 2009;38(02):285-300

35 Hornof WJ, Wind AP, Wallack ST, Schulz KS. Canine elbow dysplasia. The early radiographic detection of fragmentation of the coronoid process. Vet Clin North Am Small Anim Pract 2000; 30(02):257-266, v

36 Cook CR, Cook JL. Diagnostic imaging of canine elbow dysplasia: a review. Vet Surg 2009;38(02):144-153

37 Janach KJ, Breit SM, Künzel WW. Assessment of the geometry of the cubital (elbow) joint of dogs by use of magnetic resonance imaging. Am J Vet Res 2006;67(02):211-218

38 Preston CA, Schulz KS, Kass PH. In vitro determination of contact areas in the normal elbow joint of dogs. Am J Vet Res 2000;61(10): 1315-1321

39 Gemmill TJ, Mellor DJ, Clements DN, et al. Evaluation of elbow incongruency using reconstructed CT in dogs suffering 
fragmented coronoid process. J Small Anim Pract 2005;46(07): 327-333

40 Mason DR, Schulz KS, Samii VF, et al. Sensitivity of radiographic evaluation of radio-ulnar incongruence in the dog in vitro. Vet Surg 2002;31(02):125-132

41 Gemmill TJ, Hammond G, Mellor D, Sullivan M, Bennett D, Carmichael S. Use of reconstructed computed tomography for the assessment of joint spaces in the canine elbow. J Small Anim Pract 2006;47(02):66-74

42 Wagner K, Griffon DJ, Thomas MW, et al. Radiographic, computed tomographic, and arthroscopic evaluation of experimental radioulnar incongruence in the dog. Vet Surg 2007;36(07):691-698

43 Böttcher P, Werner H, Ludewig E, Grevel V, Oechtering G. Visual estimation of radioulnar incongruence in dogs using threedimensional image rendering: an in vitro study based on computed tomographic imaging. Vet Surg 2009;38(02):161-168

44 Samoy Y, Gielen I, Van Caelenberg A, van Bree H, Duchateau L, Van Ryssen B. Computed tomography findings in 32 joints affected with severe elbow incongruity and fragmented medial coronoid process. Vet Surg 2012;41(04):486-494

45 Starke A, Böttcher P, Pfeil I. Röntgenologische Quantifizierung der EllbogenGelenkskonformation mit einer neuen standardisierten
Röntgentechnik unter Last (Radiologic quantification of the elbow conformation with a new method for acquiring standardized $\mathrm{x}$ rays under load. Reference values for medium sized and large dogs without dysplasia of the elbow joint). Tierarztl Prax 2013; 41:145-154

46 Starke A, Böttcher P, Pfeil I. Vergleichende röntgenologische Untersuchung von kaninen Ellbogengelenken mit und ohne Ellbogen-dysplasie unter standardisierten Belastungsbedingungen (Comparative radiologic examination of the canine elbow with and without elbow dysplasia under standardized load). Tierarztl Prax 2014;42:141-150

47 Reichle JK, Park RD, Bahr AM. Computed tomographic findings of dogs with cubital joint lameness. Vet Radiol Ultrasound 2000;41 (02):125-130

48 Skinner OT, Warren-Smith CM, Burton NJ, Parsons KJ. Computed tomographic evaluation of elbow congruity during arthroscopy in a canine cadaveric model. Vet Comp Orthop Traumatol 2015;28 (01):19-24

49 Werner H, Winkels P, Grevel V, Oechtering G, Böttcher P. Sensitivity and specificity of arthroscopic estimation of positive and negative radio-ulnar incongruence in dogs. An in vitro study. Vet Comp Orthop Traumatol 2009;22(06):437-441 